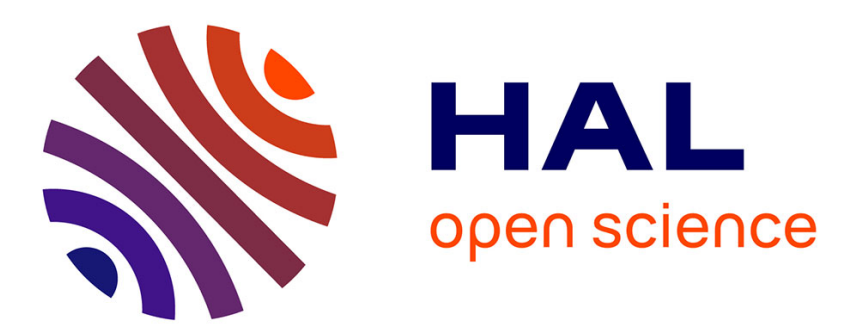

\title{
Modeling Sliding Friction Between Human Finger and Touchscreen Under Electroadhesion
}

\author{
Cagatay Basdogan, M. Reza Alipour Sormoli, Omer Sirin
}

\section{To cite this version:}

Cagatay Basdogan, M. Reza Alipour Sormoli, Omer Sirin. Modeling Sliding Friction Between Human Finger and Touchscreen Under Electroadhesion. IEEE Transactions on Haptics (ToH), 2020, 13 (3), pp.511-521. 10.1109/TOH.2020.2989221 . hal-03176779

\author{
HAL Id: hal-03176779 \\ https://hal.science/hal-03176779
}

Submitted on 22 Mar 2021

HAL is a multi-disciplinary open access archive for the deposit and dissemination of scientific research documents, whether they are published or not. The documents may come from teaching and research institutions in France or abroad, or from public or private research centers.
L'archive ouverte pluridisciplinaire HAL, est destinée au dépôt et à la diffusion de documents scientifiques de niveau recherche, publiés ou non, émanant des établissements d'enseignement et de recherche français ou étrangers, des laboratoires publics ou privés. 


\title{
Modeling Sliding Friction between Human Finger and Touchscreen Under Electroadhesion
}

\author{
Cagatay Basdogan, Member, IEEE, M.Reza Alipour Sormoli, and Omer Sirin
}

\begin{abstract}
When an alternating voltage is applied to the conductive layer of a capacitive touchscreen, an oscillating electroadhesive force (also known as electrovibration) is generated between the human finger and its surface in the normal direction. This electroadhesive force causes an increase in friction between the sliding finger and the touchscreen. Although the practical implementation of this technology is quite straightforward, the physics behind voltage-induced electroadhesion and the resulting contact interactions between human finger and the touchscreen are still under investigation. In this paper, we first present the results of our experimental study conducted with a custom-made tribometer to investigate the effect of input voltage on the tangential forces acting on the finger due to electroadhesion during sliding. We then support our experimental results with a contact mechanics model developed for estimating voltage-induced frictional forces between human finger and a touchscreen as a function of the applied normal force. The unknown parameters of the model were estimated via optimization by minimizing the error between the measured tangential forces and the ones generated by the model. The estimated model parameters show a good agreement with the ones reported in the literature.
\end{abstract}

Index Terms—surface haptics, contact mechanics, electroadhesion, electrovibration, friction, and contact area.

\section{INTRODUCTION}

C URRENTLY, there are mainly three different technologies for detecting finger position on a touchscreen: resistive, capacitive, and surface acoustic wave. Among those three, the capacitive touchscreens are the most popular because of their several advantages over the other two. In fact, the launch of Apple's iPhone in 2007 made the public to refocus on them as never before. The capacitive touchscreens are two types: surface and projected. A surface capacitive touchscreen utilizes a single layer of conductive ITO with a simple electrode pattern on top of it and passing through its edges. This layer is placed above a glass panel and covered by an insulating coating $\left(\mathrm{SiO}_{2}\right)$. When a finger contacts the screen, some of the electrical charges transfer from the screen to the finger. Sensors in all four corners of the screen detect the decrease in electric current. The controller then determines the touch point via interpolation. Rather than a sensor, a surface capacitive touchscreen can be also utilized as an actuator to display tactile effects to the human finger. When an alternating voltage is applied to the conductive layer of a surface capacitive touchscreen, an electrostatic attractive force is generated between the finger and the surface in the normal direction, which results in an increase in frictional forces acting on the finger sliding on the surface in the tangential direction. By altering the amplitude, frequency, and waveform of this signal, a rich set of tactile effects can be generated on the touchscreen. Despite the ease of implementation, the contact mechanics leading to an increase in frictional forces in tangential direction due to voltage-induced electrostatic forces in normal direction has not been fully understood yet.

- C. Basdogan, M. Alipour, and O. Sirin are with the Department of Mechanical Engineering, Koc University, Istanbul 34450, Turkey.

E-mail: \{cbasdogan, msormoli18, osirin13\}@ku.edu.tr

\# Corresponding author: cbasdogan@ku.edu.tr

Manuscript received XX XX, 2019; revised XX XX, 2019.
In order to estimate the electrostatic force, $F_{e}$, between finger pad and touchscreen, models based on parallel-plate capacitor theory have been proposed (see the summary of models in [1]). These models assume a constant air gap between two surfaces and ignore the effect of asperities on both surfaces. On the other hand, to estimate the frictional forces in tangential direction during sliding, the Coulomb model of friction utilizing a constant coefficient of sliding friction between finger pad and touchscreen has been used so far. The increase in tangential force has been explained by simply adding the force due to electrostatic attraction to the normal force applied by the finger, $F_{t}=\mu\left(F_{n}+F_{e}\right)$, where $\mu$ is the sliding friction coefficient, $F_{t}$, and $F_{n}$, represent the tangential and normal forces applied to the finger, respectively. However, it is already known that friction of human finger pad against smooth surfaces is governed by adhesion, which results in a nonlinear relation between friction coefficient and the normal force. The frictional force due to adhesion can be calculated by $F_{t}=\tau A_{\text {real }}$, where $\tau$ is the interfacial shear stress and $A_{\text {real }}$ is the real area of contact. It is highly difficult to measure or estimate the real area of contact, which is only a small fraction of the apparent contact area, $A_{a p p}$, and varies nonlinearly with the normal force. Moreover, in the case of electroadhesion, there is a coupling between the voltage-induced electric field and the mechanical forces acting on the finger and the nature of this interaction has not been fully exploited yet. For example, there is no feeling of electrostatic forces when the finger is stationary and the reason behind is still under investigation. Shultz et al. [2] showed that the electrical impedance of the interfacial gap between finger and touchscreen is significantly lower for the stationary finger compared to that of the sliding finger under electroadhesion, suggesting the important role of air gap and finger moisture. Perhaps, the magnitude of the electroadhesive force is reduced when the finger is stopped as sweat accumulates in the interfacial 
gap and shorts out any voltage applied. Ayyildiz et al. [3] and Sirin et al. [4] speculated that the main cause of the increase in friction under electroadhesion is an increase in real contact area due to a change in air gap. They investigated the sliding friction as a function of normal force and the voltage amplitude using a mean field theory based on multiscale contact mechanics and showed that the interfacial gap affects the size of relative contact area $A_{\text {real }} / A_{\text {app }}$. They argued that the voltage-induced electrostatic forces cause the finger asperities make more contacts with the surface of touchscreen at microscopic scale. In fact, this claim originates from Persson's sliding friction theory [5], [6], which suggests that the real contact area for elastic surfaces (such as human finger) having different length scales is significantly larger than it was originally thought for increasing normal load. Each asperity making microscopic contact can further support adhesive shear load proportional to its own contact area, increasing the overall magnitude of tangential forces shearing those contacts.

In this paper, we first present the results of our experimental study conducted with a custom-made tribometer to investigate the effect of input voltage on the tangential forces acting on the finger under electroadhesion. These experiments provide significant empirical data and fresh insight into the contact mechanics between fingerpad and touchscreen. We support our experimental results with a contact mechanics model developed for estimating voltageinduced frictional forces between human finger and a touchscreen as a function of the normal force. In our model, the tangential frictional force due to electroadhesion is simply calculated using $F_{t}=\tau A_{\text {real }}$. Compared to our earlier modeling studies [3], [4], we estimate the interfacial shear stress, $\tau$, using the adhesive friction model of Adams et al. [7] and the real contact area, $A_{\text {real }}$, as a fraction of the apparent contact area, which is calculated by the well-known JKR contact model [8]. The fraction coefficient is assumed to be a linear function of the electroadhesive energy, which is estimated by the parallel-plate capacitor model for a given input voltage amplitude. The unknown parameters of the proposed model were estimated via optimization by minimizing the error between the measured tangential forces and the ones generated by the model. The estimated model parameters show a good agreement with the ones reported in the literature.

The rest of this article is organized as follows: Section 2 presents the theory behind our contact model. The experimental set-up and procedure for the contact mechanics experiments are given in Section 3. Sections 4 reports the experimental results and comparisons made with the theoretical model. Discussion of the results and some conclusions are provided in Section 5 .

\section{THEORY}

In order to explain the concept of contact interactions between a finger and a touchscreen under electroadhesion, we first consider a parallel-plate capacitor model and then estimate the electrostatic attractive force between the plates when there is a potential difference, $V$, between them. A capacitance exists whenever two electrodes are separated by some distance. Capacitance $C$ is the measure of the charge

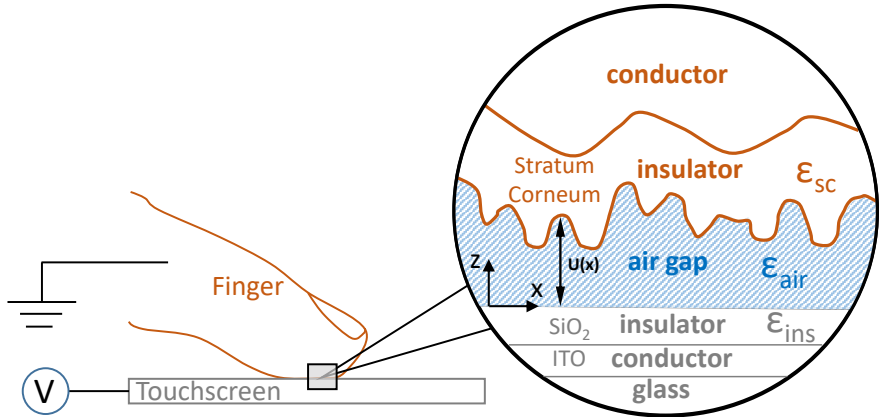

Fig. 1. The interface between human finger and touchscreen under electroadhesion. A surface capacitive touchscreen is made of 3 layers: an insulating coating $\left(\mathrm{SiO}_{2}\right)$ at the top, a conductive (ITO) layer in the middle, and a glass panel at the bottom. Though it is more complex, the human finger can be considered to be made of 2 layers: an insulating layer (stratum corneum) and a conductive layer below it. Due to the finger prints, there is always some air gap between fingerpad and touchscreen even when finger is fully pressed to the screen.

$Q$ (i.e., number of electrons) that a capacitor holds when a certain electric potential $V$ is applied: $C=Q / V$. The work needed to store a total charge $Q$ in a capacitor is:

$$
W=\int_{0}^{Q} V d q=\frac{1}{C} \int_{0}^{Q} q d q=\frac{1}{2} \frac{Q^{2}}{C}
$$

Using $Q=C V$,

$$
W=\frac{1}{2} C V^{2}
$$

This work is stored as potential energy in the capacitor. We can obtain the magnitude of electrostatic force, $F_{e}$, by calculating the derivative of the work along the direction in which the work is done, and using $C=\left(\varepsilon_{a i r} \varepsilon_{0} A\right) / z$, where, $z$ is the variable distance between the plates, $\varepsilon_{a i r}$ is the relative permittivity of the air, $\varepsilon_{0}$ is the electric permittivity of vacuum, and $A$ is the overlapping area of the plates.

$$
F_{e}=\left|\frac{d W}{d z}\right|=\left|\frac{d W}{d C}\right|\left|\frac{d C}{d z}\right|_{z=d}=\varepsilon_{a i r} \varepsilon_{0} \frac{A V^{2}}{2 d^{2}}
$$

where, $d$ is the fixed distance between the plates.

In the case of modeling interactions between human finger and touchscreen (see Fig. 1), one needs to consider their multi-layered structure. In addition to the top insulating layer of the touchscreen $\left(\mathrm{SiO}_{2}\right)$, the outermost layer of human epidermis (stratum corneum) as well as the air gap between them also act as insulators. Hence, the voltage difference between the conductive layers of human finger and the touchscreen can be written as $V=V_{\mathrm{SiO}_{2}}+V_{\text {air }}+V_{S C}$. Assuming no leakage of charge, the effective capacitance of the system can be calculated as three capacitances in series:

$$
\begin{aligned}
C & =\frac{1}{\left(\frac{1}{C_{S_{i O}}}+\frac{1}{C_{a i r}}+\frac{1}{C_{S C}}\right)} \\
& =\frac{\varepsilon_{0} A_{a p p}}{\left(\frac{d_{S_{i O}}}{\varepsilon_{S_{i O}}}+\frac{z}{\varepsilon_{a i r}}+\frac{d_{S C}}{\varepsilon_{S C}}\right)}
\end{aligned}
$$


where, $A_{a p p}$ is the apparent contact area of fingerpad, $d_{\mathrm{SiO}_{2}}$ and $d_{S C}$ represent the thicknesses of the insulator layer of touchscreen and stratum corneum, $\varepsilon_{\mathrm{SiO}_{2}}$ and $\varepsilon_{\mathrm{SC}}$ are their relative permittivities, respectively. Here, we assume that those thicknesses are constant while the thickness of the air gap varies in $\mathrm{z}$-direction with respect to $\mathrm{x}$ (see Fig. 1) due to the finger surface roughness, and also depending on the normal force applied by the finger on the touchscreen. The electrostatic attractive force between human finger and touchscreen can be calculated from the work for a constant air gap as:

$$
\begin{aligned}
F_{e} & =\left|\frac{d W}{d z}\right|_{z=d_{a i r}} \\
& =\varepsilon_{0} \frac{A_{a p p} V^{2}}{2\left(\frac{d_{S i O_{2}}}{\varepsilon_{S i O_{2}}}+\frac{d_{a i r}}{\varepsilon_{a i r}}+\frac{d_{S C}}{\varepsilon_{S C}}\right)^{2} \varepsilon_{a i r}}
\end{aligned}
$$

where, $d_{\text {air }}$ is the mean thickness of the air gap for the applied normal force.

Now, we will have a close look at the individual parameters contributing to the electrostatic force. First of all, the electrostatic force is proportional to the square of the applied voltage, and hence the relation between them is nonlinear. Due to this relation, period doubling occurs in the electrostatic force response when an alternating voltage is applied to the conductive layer of touchscreen (e.g. if the frequency of the input voltage is $125 \mathrm{~Hz}$, then the frequency of the output electrostatic force is $250 \mathrm{~Hz}$ ). Here, the relative permittivity of the air can be taken as one $\left(\varepsilon_{\text {air }}=1.0005\right.$ at 20 degrees Celsius). The relative permittivity of the insulator layer of the touchscreen does not vary much with frequency $\left(\varepsilon_{\mathrm{SiO}_{2}} \approx 3.9\right)$ and its magnitude is small compared to that of the stratum corneum, which varies with the frequency of stimulation $\left(\varepsilon_{S C}\right.$ varies approximately between $10^{4}$ and $10^{3}$ for the frequency varying from $10 \mathrm{~Hz}$ to $1000 \mathrm{~Hz}$ ). Moreover, the ratio of $\left(d_{\mathrm{SiO}_{2}} / \varepsilon_{\mathrm{SiO}_{2}}\right)$ is approximately 2075 times larger than $\left(d_{S C} / \varepsilon_{S C}\right)$ since the thickness of the insulator for a typical touchscreen is around $d_{\mathrm{SiO}_{2}}=1 \mu \mathrm{m}$ while that of the stratum corneum is $d_{S C} \approx 10-40 \mu \mathrm{m}$. The mean thickness of the interfacial air gap, $d_{a i r}$, though it is small, is an important parameter effecting the magnitude of electrostatic force. This gap varies with the normal force applied by the finger and difficult to measure. However, if we, for example, take $\varepsilon_{S C} \approx 3000$ for the frequency of $250 \mathrm{~Hz}$ [9], $d_{S C}=25 \mu m$, then an air gap thickness that is significantly larger (smaller) than the threshold value of $d_{0}=\left(d_{\mathrm{SiO}_{2}}\right) /\left(\varepsilon_{\mathrm{SiO}_{2}}\right)+\left(d_{S C}\right) /\left(\varepsilon_{S C}\right) \approx 265 \mathrm{~nm}$ will reduce (increase) the magnitude of electrostatic force. Another parameter in Eq. 5 is the apparent area of finger contact, $A_{a p p}$, which varies nonlinearly with the applied normal force (see a review in [10]). The apparent contact area for a stationary finger can be estimated using the JKR theory as:

$A_{\text {app }}^{0}=\pi\left[\frac{3 R}{4 E^{*}}\left(F_{n}+3 \Delta \gamma \pi R+\sqrt{6 \Delta \gamma \pi R F_{n}+(3 \Delta \gamma \pi R)^{2}}\right)\right.$

where, $R$ is the radius of curvature for the fingerpad- touchscreen interface and $\Delta \gamma$ is the work done by electroadhesion, $E^{*}$ is the effective elastic modulus, which is reduced to $E^{*}=\left(E_{\text {finger }}\right) /\left(1-\nu_{\text {finger }}^{2}\right)$ since the bulk elastic modulus of fingerpad, $E_{\text {finger }}$, is much lower than that of the touchscreen (note that the Poisson ratio for fingerpad, $\nu_{\text {finger, }}$, can be taken as 0.5).

The work done by electroadhesion, $\Delta \gamma$, can be calculated by [11]

$$
\Delta \gamma=\int_{d_{\text {air }}}^{\infty} p_{e} d z
$$

where, $p_{e}=F_{e} / A_{a p p}$ is the average normal pressure due to electroadhesion. If the above integral is calculated using the given lower and upper bounds, we obtain

$$
\Delta \gamma=\frac{1}{2} \varepsilon_{0} V^{2}\left(\frac{d_{S i O_{2}}}{\varepsilon_{S i O_{2}}}+\frac{d_{a i r}}{\varepsilon_{a i r}}+\frac{d_{S C}}{\varepsilon_{S C}}\right)^{-1}
$$

Using the values of $d_{\mathrm{SiO}_{2}}=1 \mu \mathrm{m}, d_{\mathrm{SC}}=25 \mu \mathrm{m}, d_{\text {air }}=$ $1.0 \mu \mathrm{m}, \varepsilon_{\mathrm{SiO}_{2}} \approx 3.9, \varepsilon_{S C} \approx 3000$ at $250 \mathrm{~Hz}$, and voltage amplitude of $V=100$ volts, the work of electroadhesion can be estimated as $\Delta \gamma \approx 0.035 \mathrm{~J} / \mathrm{m}^{2}$.

Returning back to the JKR model given in Eq. 6, if the adhesion work is zero, then it is reduced to the wellknown Hertz contact model and the contact area can be calculated as $A_{H}=\pi\left[3 R F_{n} /\left(4 E^{*}\right)\right]^{2 / 3}$. On the other hand, if the external normal force is set to zero $\left(F_{n}=0\right)$, then one can calculate the contact radius for pull-off, $r^{\text {pull-off }}=$ $\left[9 \pi R^{2} \Delta \gamma /\left(2 E^{*}\right)\right]^{1 / 3}$. Using the pull-off radius, one can also calculate the normal force to pull the finger off from the surface of touchscreen as

$$
F_{n}^{\text {pull-off }}=-\frac{3}{2} \Delta \gamma \pi R
$$

Here, if we assume that radius of curvature is $R \approx 20 \mathrm{~mm}$ for a contact angle of 45 degrees (see the supplementary material in [12]), then the pull-off force to separate the finger from the touchscreen in the normal direction is $F_{n}^{\text {pull-off }} \approx 3.3 \mathrm{mN}$ based on the electroadhesive work estimated as $\Delta \gamma \approx 0.035 \mathrm{~J} / \mathrm{m}^{2}$ for a voltage amplitude of $V=100$ Volts. This estimation is in line with the values reported in earlier experimental studies without electroadhesion. Pailler-Mattei et al. [13] reported a maximum pulloff force of $5 \mathrm{mN}$ between human skin and a custum-made tribometer having a spherical steel indenter with a radius of curvature of $R=6.35 \mathrm{~mm}$. Spinner et al. [14] report that human fingers can generate a maximum adhesive force of $15 \mathrm{mN}$ on a smooth surface of epoxy resin. As can be seen from the above analysis, it is easy to break the bonds due to electroadhesion in the normal direction. This also explains why the electrostatic forces due to electroadhesion are not perceivable when the finger is stationary.

${ }^{2 / 3}$ Now, using Eq. 6, we can also estimate the apparent and pull-off contact areas for a typical normal force of $F_{n}=1 \mathrm{~N}$. If we assume a bulk elastic modulus of $E_{\text {finger }}=45 \mathrm{kPa}$ for the fingerpad, then we obtain $A_{a p p}^{0} \approx 133 \mathrm{~mm}^{2}$ $\left(r_{a p p}^{0}=6.5 \mathrm{~mm}\right), A_{H}=125 \mathrm{~mm}^{2}\left(r_{H}=6.3 \mathrm{~mm}\right)$, and 
$A^{\text {pull-off }}=7 \mathrm{~mm}^{2}\left(r_{H}^{\text {pull-off }}=1.5 \mathrm{~mm}\right)$. The values estimated for the apparent area using JKR and Hertz models are again compatible with the experimental values reported in the earlier studies [10].

The apparent contact area estimated by Eq. 6 is for the case in which only normal stress acts on the contact interface and there is no tangential loading. A recent study [15] shows that the apparent finger contact area reduces during frictional contacts due to the tangential loading even when there is no electroadhesion. Since electroadhesion increases the friction and hence the tangential load, one expects a further reduction in the apparent finger contact area, as reported in Sirin et al. [16]. They conducted an experimental study to investigate the contact evolution between the human finger and a touchscreen under electroadhesion using a robotic set-up and an imaging system. The results show that the effect of electroadhesion is only present during full slip but not before slip. The coefficient of friction increases under electroadhesion as expected during full slip, but the apparent contact area is significantly smaller during full slip when compared to that of no electroadhesion condition and the amount of reduction depends on the tangential load. The authors suggest that the reduction in apparent area is due to stiffening of the finger skin in the tangential direction.

Modeling the adhesive elastic contacts under both normal and tangential loading is highly challenging. As stated in [17], a general theory of adhesion under superimposed normal and tangential loads does not exist even in the case of purely elastic contacts since the energy balance method used in JKR model loses its applicability when nonconservative forces causing an energy dissipation, such as the frictional force, exists at the interface. This also explains why the contact models based on fracture-mechanics have been developed to capture the effects of energy dissipation in adhesive contact problems under tangential loading [18], [19].

Thus, in our study, in order to estimate the apparent contact area for sliding finger under tangential loading, $A_{a p p}$, we rely on the empirical model developed by [15]:

$$
A_{\text {app }}=A_{a p p}^{0}-\eta F_{t}^{2}
$$

where, $A_{a p p}^{0}$ is the initial apparent area for the stationary finger (no sliding) and calculated by Eq. 6 in our approach and $\eta$ is a free parameter, which we estimate through an optimization, as further detailed in Section 4.

Now, if the real contact area, $A_{\text {real }}$, is assumed to be a small fraction of the apparent contact area, $A_{a p p}$, the frictional force due to electroadhesion can be calculated by using $F_{t}=\tau A_{\text {real }}$. For the interfacial shear stress, $\tau$, Adams et al. [7] adopted a model from an early study by Bowden and Tabor [20], which suggests that $\tau$ increases linearly with the mean contact pressure as $\tau=\tau_{0}+\alpha p_{e}$, where $\tau_{0}$ is the intrinsic shear strength and $\alpha$ is the pressure coefficient. Hence, the electroadhesive friction can be expressed as:

$$
F_{t}=\tau_{0} A_{\text {real }}+\alpha F_{n}
$$

The first term is more dominant at small normal forces where the electroadhesion is effective while the latter one

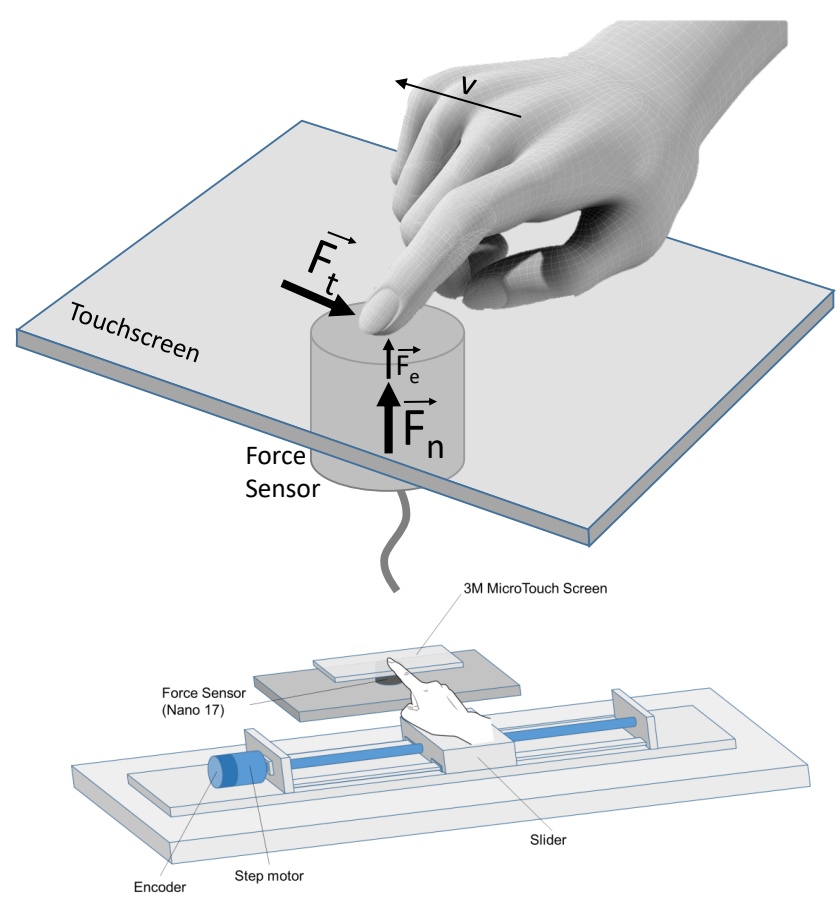

Fig. 2. The experimental setup used to measure the force response of the experimenter's index finger in the normal and tangential directions via a force transducer placed under the touchscreen. The experimenter's hand was placed on the slider, driven by a step motor, such that the phalanges of the index finger were aligned with an angle of approximately 30 degrees with respect to the touchscreen, and the tip of the index finger was always in contact with the touchscreen during the forward and backward translation of the slider along the horizontal direction.

represents the steady-state sliding friction at higher normal forces [21]. Here, we assume a relation of $A_{\text {real }}=\beta A_{\text {app }}$, where $\beta<1$ is the ratio of real contact area to the apparent contact area. The results of our recent studies [3], [4] show that electroadhesion causes an increase in the real contact area at the miscroscopic level with increasing input voltage. Hence, one expects that the coefficient $\beta$ increases with the electroadhesive energy. In this study, we assume a linear relation between the area ratio and the electroadhesive energy in the form of $\beta=\beta_{0}+\varphi \Delta \gamma$, where $\beta_{0}$ is the nominal value of area ratio when there is no electroadhesion and $\varphi$ is a free parameter which we estimate through an optimization, as further detailed in Section 4. Then, the tangential force can be written as [22];

$$
F_{t}=\tau_{0}\left(\beta_{0}+\varphi \Delta \gamma\right) A_{a p p}+\alpha F_{n}
$$

As obvious from Eq. 12, the tangential force is not zero and equal to $F_{t}^{\text {slip }}=\tau_{0} \beta A_{\text {app }}$ even when there is no external normal load.

\section{Methods}

\subsection{Experimental Set-Up}

We designed and built a custom-made tribometer in order to measure tangential force acting on the human finger during sliding as a function of normal force for different input voltages applied to a touchscreen. The major components 
of our tribometer include a high-torque step motor moving a slide on a power screw and a force sensor (Nano 17, ATI Industrial Automation Inc.) attached to the base of the touchscreen (SCT-3250, 3M Inc.). The step motor (MDrive23Plus, Intelligent Motion Systems Inc.) was commanded to translate the slide back and forth in horizontal direction at a desired velocity. The experimenter's hand was placed on the slide such that his finger was always in contact with the touchscreen during the sliding. As the experimenter's finger was moving on the touchscreen, the force response was measured using the force transducer placed under the touchscreen. The normal and tangential forces were acquired by a 16-bit analog data acquisition card (PCI-6034E, National Instruments Inc.) with a sampling rate of $10 \mathrm{kHz}$.

\subsection{Experimental Procedure}

The experiment required the control of normal force manually, which was achieved by visual tracking of the force magnitude from a large screen oscilloscope after some training. Due to the large number of trials and manual control of normal force, the experiment was conducted with a single and well-trained experimenter. Before the experiment, the experimenter washed his hands with soap, rinsed with water, and dried them in room temperature. Moreover, the touchscreen was cleaned by alcohol before the experiment. The experimenter worn a ground strap on his stationary wrist. The study was approved by the ethics committee at Koc University. The investigation conformed to the principles of the Declaration of Helsinki and the experiment was performed in accordance with relevant guidelines and regulations. A sinusoidal voltage signal at frequency of 125 $\mathrm{Hz}$ with each of the following amplitudes of $25 \mathrm{~V}, 50 \mathrm{~V}, 75$ $\mathrm{V}, 100 \mathrm{~V}, 125 \mathrm{~V}, 150 \mathrm{~V}, 175 \mathrm{~V}$, and $200 \mathrm{~V}$ was applied to the touchscreen. The finger of the experimenter moved back and forth on the touchscreen (one cycle) for a total displacement of $80 \mathrm{~mm}$ at a sliding velocity of $50 \mathrm{~mm} / \mathrm{s}$. As the experimenter's finger was moving on the touchscreen, the force response was measured using the force transducer. For each applied voltage, the experimenter aimed to increase his normal force from $0.2 \mathrm{~N}$ to $2 \mathrm{~N}$ with an increment of $0.2 \mathrm{~N}$ after every other 4 cyles. The electrovibration was turned on $(E V=O N)$ and off $(E V=O F F)$ after every other cycle. Hence, the experimenter made a total number of 320 cycles (8 different voltages $\times 10$ increments of normal force per applied voltage $x 4$ cycles per force increment); resulting in 160 cycles for each experimental condition (i.e., $E V=O F F$ and $E V=O N)$. The measured force data was filtered by a low pass filter having a cut-off frequency of $500 \mathrm{~Hz}$. The cut-off frequency was selected by considering the frequency limits of human vibrotactile perception. Only the force data corresponding to a travel length of $10 \mathrm{~mm}$, centered around the force sensor, was processed for the analysis.

\section{Results}

\subsection{Curve-Fitting to the Experimental Data}

For curve-fitting to the experimental data, Eq. 12 can be written in the following simplified form:

$$
F_{t}=a+b F_{n}^{c}+d F_{n}
$$

where, $a=F_{t}^{s l i p}, b, c$, and $d=\mu_{s s}$ (steady-state friction coefficient) are constant parameters that can be estimated from the curve fit. In order to fit a curve to the experimental data and obtain a unique set of parameters, we eliminate the small constant term in Eq. 13 to obtain:

$$
F_{t}=b F_{n}^{c}+d F_{n}
$$

In the above equation, the nonlinear first term represents the neck observed at low normal forces while the linear term is more effective when the normal forces are high and the relation between tangential friction force and normal force is linear (see Fig. 3a). Note that the above empirical model (Eq. 14) predicts the tangential force at zero normal load as zero, which does not agree with the JKR adhesion model
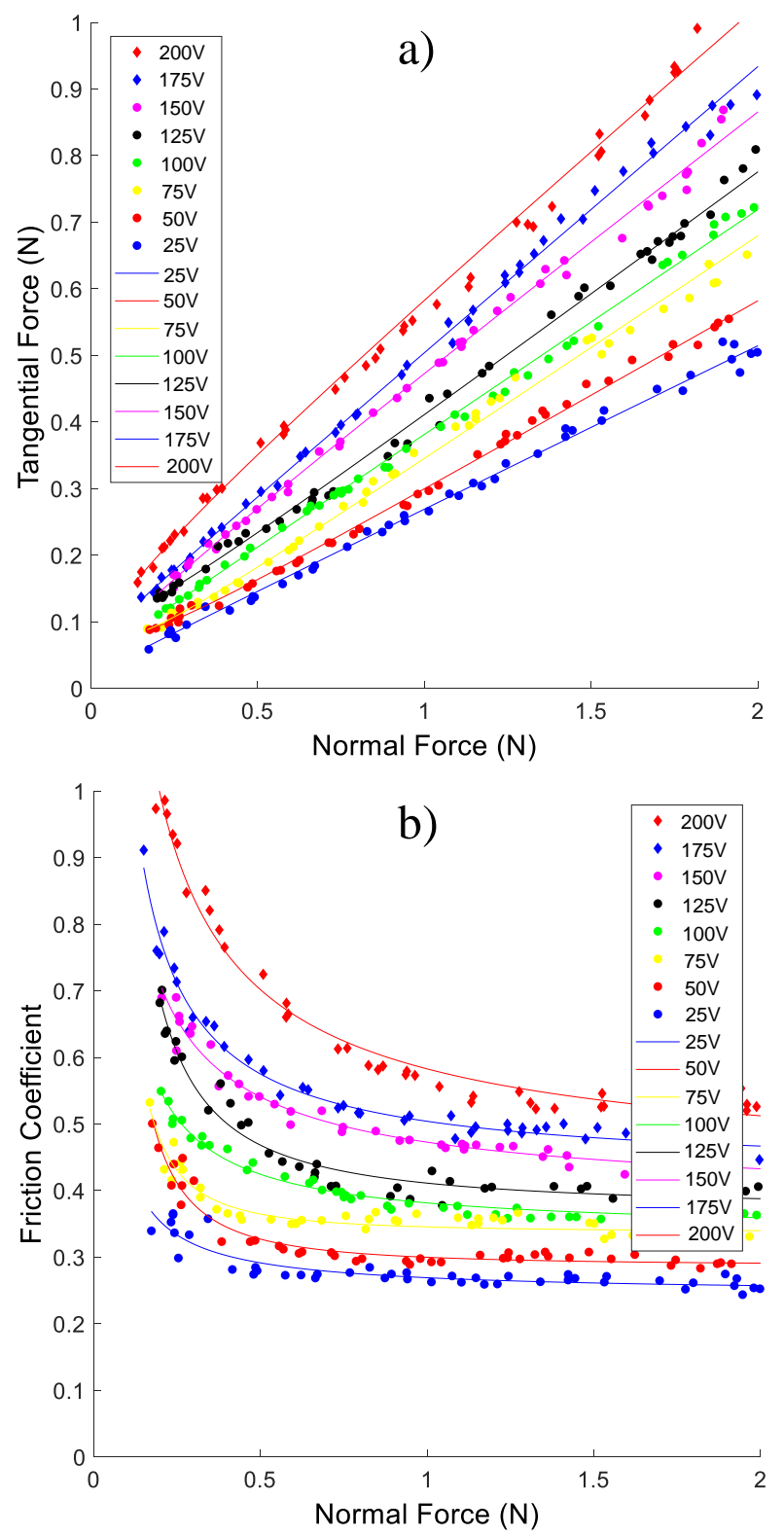

Fig. 3. Results of curve-fitting: a) tangential force versus normal force and b) friction coefficient versus normal force for different voltages applied to the touchscreen. 
TABLE 1

The list of parameters and their values used in the contact mechanics model

\begin{tabular}{|c|c|c|c|c|c|}
\hline Fixed & Optimized & Definition & Range & Value & Unit \\
\hline$\varepsilon_{S_{i O}}, \varepsilon_{S C}$ & & Relative permittivities of $\mathrm{SiO}_{2}$ and $\mathrm{SC}$ & & $3.9,3 \times 10^{3}($ at $250 \mathrm{~Hz})$ & \\
\hline$d_{\mathrm{SiO}_{2}}, d_{\mathrm{SC}}$ & & Thicknesses of $\mathrm{SiO}_{2}$ and $\mathrm{SC}$ & & $1 \times 10^{-6}, 25 \times 10^{-6}$ & $m$ \\
\hline$R$ & & Radius of curvature & & $2 \times 10^{-2}$ & $m$ \\
\hline \multirow[t]{7}{*}{$\alpha$} & & Pressure coefficient & & $\begin{array}{l}\text { taken from the exper- } \\
\text { imental data as the } \\
\text { steady-state value of the } \\
\text { sliding friction coeffi- } \\
\text { cient for each input } \\
\text { voltage ( } \alpha=\mu_{s s} \text {, see } \\
\text { Fig. 5a) }\end{array}$ & \\
\hline & $E^{*}$ & Effective elastic modulus & $2.0-100$ & 35.00 & $k P a$ \\
\hline & $\tau_{0}$ & Interfacial shear strength & $0.0-15.0$ & 10.64 & $k P a$ \\
\hline & $d_{a i r}$ & Mean thickness of the airgap & $0.0-5.0$ & $190 \times 10^{-3}$ & $\mu m$ \\
\hline & $\beta_{0}$ & $\begin{array}{l}\text { Initial value of the area ratio }\left(A_{\text {real }} / A_{a p p}\right) \\
\text { when } E V=O F F\end{array}$ & $0.0-1.0$ & 0.0049 & \\
\hline & $\varphi$ & Constant coefficient for adhesive energy & $0.0-1.0$ & 0.47 & \\
\hline & $\eta$ & Constant coefficient for sliding contact area & $0.0-1.0$ & $3.72 \times 10^{-4}$ & $m^{2} / N^{2}$ \\
\hline
\end{tabular}

given in Eq. 12. Nevertheless, compared to the other terms, the non-zero term is small and more importantly, dropping this term leads to unique fit of this empirical model to the experimental data of friction coefficient versus normal force. For this purpose, we first divide the left and right hand sides of Eq. 14 by the normal force term to obtain a relation between friction coefficient and normal force as:

$$
\mu=b F_{n}^{c-1}+d
$$

Then, we fit Eq. 15 to the experimental data of friction coefficient versus normal force to obtain a unique set of constant parameters $(b, c, d)$. The results of our empirical fit for tangential force and friction coefficient for different input voltages are shown in Fig. 3. As it is clear from these plots, the magnitude of tangential force and friction coefficient increase as the input voltage amplitude is increased. The relative increase in steady-state friction (coefficient " $\mathrm{d}$ " in Eq. 15) as a function of voltage amplitude is shown in Fig. 4a. A linear curve fit reveals that the steady-state friction increases by $0.25 \%$ per volt (Fig. $4 b$ ). Hence, for example, the relative increase in sliding friction is $25 \%$ for a sinusoidal voltage with an amplitude of $100 \mathrm{~V}$ and frequency of 125 $\mathrm{Hz}$ is applied to the screen.

\subsection{Estimation of Electrostatic Force from the Experi- mental Data}

While it is highly difficult to measure the electrostatic force in the normal direction using a force sensor directly, it can be inferred from the measured tangential and normal forces acting on the finger during sliding [23]. Using the curves of $E V=O F F$ and $E V=O N$ conditions for different voltages, we estimated the magnitude of the electrostatic forces as a function of normal force.

When the normal force applied by human finger under $E V=O F F$ and $E V=O N$ conditions is the same (Fig. 5a), the corresponding tangential forces becomes
$F_{t}^{O F F}=\mu^{O F F} F_{n}$ and $F_{t}^{O N}=\mu^{O N} F_{n}$, respectively. Here, we consider the fact that the friction coefficient changes under $E V=O N$ condition and the relative increase in tangential force $\left(\Delta F_{t}\right.$ in Fig. $\left.5 \mathrm{~b}\right)$ is due to the electrostatic force times the new friction coefficient, $F_{t}^{O N}=F_{t}^{O F F}+\mu^{O N} F_{e}$. As a result, the electrostatic force can be inferred from the experimental data (Fig. 5c) using the following relation:

$$
F_{e}=\left(1-\frac{\mu^{O F F}}{\mu^{O N}}\right) F_{n}=\left(\frac{\Delta \mu}{\mu^{O N}}\right) F_{n}
$$

A slightly different approach was followed by Meyer et al. [23] to estimate the electrostatic forces $\left(F_{e}=\Delta F_{t} / \mu\right)$ by assuming that the friction coefficient does not change when $E V=O N$, which is not the case as reported in Fig. 3b. Moreover, the dependency of electrostatic force on the applied normal force has been ignored in their approach and the electrostatic force was calculated for one value of normal force only $\left(F_{n}=0.5 N\right)$, but Fig. $5 d$ shows the strong dependency of electrostatic force on normal force.

\subsection{Comparison of the Experimental Data with the Model}

We estimate the model parameters via optimization (see Table 1). The goal of the optimization is to estimate the unknown parameters $E^{*}, \tau_{0}, \beta_{0}, \varphi$, and $\eta$ of the model by minimizing the error between the measured tangential force and the one generated by the model for the normal force varying between 0.2 and $2 N$, with increments of 0.01 $N$. Hence, the error function to be minimized, $E_{\text {min }}$, can be defined as:

$$
E_{\text {min }}=\sum_{i}^{N} \sum_{j}^{M}\left(F_{t}^{M O D}-F_{t}^{E X P}\right)^{2}
$$

where, $F_{t}^{M O D}$ and $F_{t}^{E X P}$ represent the tangential force estimated from the model (Eq. 12) and the one obtained from 
the experimental data via curve fitting (Eq. 14), respectively. Instead of using the raw experimental data in the optimization process, we preferred the curve-fitted values to reduce the effect of noisy measurements (see Section 4.1). $N=4$ represents the number of data sets corresponding to four different voltages (tangential force data for 50, 100, 150, 200 Volts were used only in the optimization process to reduce the computational time) and $M=200$ is the number of data points used in each data set. Fig. 6 presents the results of the optimization process. Using the optimized parameters $\left(E^{*}\right.$, $\left.\tau, \beta_{0}, \varphi, \eta\right)$ tabulated in Table 1, first, the tangential forces for each input voltage was estimated by Eq. 12 as the normal force was varied between 0.2 and $2 \mathrm{~N}$, with increments of $0.01 N$ (Fig. 6a) and then the friction coefficient was calculated by dividing the estimated tangential force values with the normal force values (Fig. 6b).

\section{Discussion ANd Conclusion}

We investigated the sliding finger friction on a smooth touchscreen under electroadhesion, induced by a voltage signal applied to its conductive layer. This voltage generates
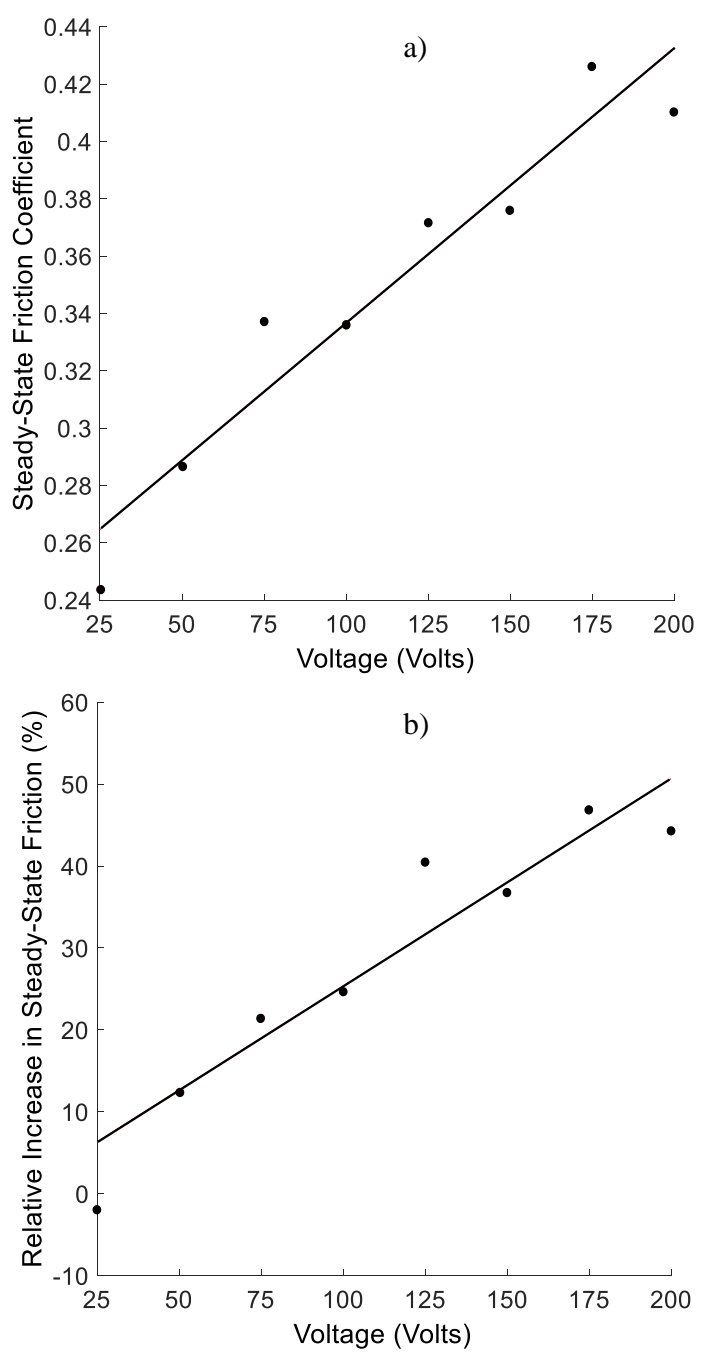

Fig. 4. a) Steady-state friction as a function of applied voltage amplitude follows a linear trend. b) Percent increase in steady-state friction as a function of applied voltage amplitude $\left(y \approx 0.25 x, R^{2}=0.9\right)$. electrostatic attractive force between the touchscreen and the finger sliding on its surface, resulting in an increase in friction applied to the finger. Using a force sensor placed under the touchscreen, we measured the tangential friction force for a range of normal forces applied by the finger on the touchscreen under different voltage amplitudes. We fit a nonlinear curve to the experimental data of friction coefficient versus normal force to estimate the magnitude of electrostatic forces causing an increase in friction. The results show that the contact is driven mainly by adhesion as suggested by the other researchers in the tribology literature for finger-smooth glass interaction [24]. Considering the fact that frictional forces due to adhesion is a nonlinear function of contact area, it is not surprising to see that the magnitude of electrostatic force changes with the applied normal force in our study. For example, the magnitude of these forces in our measurements changed from approximately 120 to $670 \mathrm{mN}$ as the normal force was increased from 0.2 to $2.0 \mathrm{~N}$ for the input voltage amplitude of 150 Volts. These values are comparable to the constant value of $250 \mathrm{mN}$ estimated by Meyer et al. [23] for $F_{n}=0.5 \mathrm{~N}$ and voltage amplitude of 140 Volts. Our results show strong dependency of electrostatic force on the applied normal force, which has not been reported before. As the normal force is increased beyond $2 N$, the electrostatic force is expected to increase, but saturate at some value eventually since the contact will get stiffer and there will not be any further increase in the apparent contact area.

In this study, we also presented a contact model to interpret the outcomes of our experimental study. In this model, the frictional force due to adhesion was calculated by $F_{t}=\tau A_{\text {real }}$, where $\tau$ was estimated by $\tau=\tau_{0}+\alpha p_{e}$ [7], [20]. The real contact area, $A_{\text {real }}$, was estimated from the sliding apparent contact area, $A_{a p p}$, using the well-known JKR contact model [8]. This model includes the effect of adhesion energy on the deformation of an elastic sphere in contact with an elastic half space. In this regard, we first estimated the adhesion energy due to induced electric field and then the JKR apparent contact area for stationary finger. As the recent experimental studies [15], [16] show, starting from the value for stationary finger, the apparent contact area for sliding finger decreases as the tangential force increases until it reaches to a steady state value (see Eq. 10). We assumed that the real contact area is only a fraction of the apparent contact area during sliding and the fraction coefficient is a linear function of the electroadhesive energy. This assumption is based on the outcomes of our recent studies [3], [4], supported by the Persson's contact theory [5], [6].

We estimated the unknown parameters of the contact model through a numerical optimization performed in Matlab, which simply minimized the error between the measured tangential force and the one generated by the contact mechanics model. Our approach is a local optimization since it finds the optimal values of the unknown parameters for the range of values given in Table 1. The range of values for $E^{*}$ and $\tau_{0}$ were selected based on the earlier experimental studies, which are cited and discussed below in detail. The ratio of real contact area to the apparent one was constrained to be less than one, $\beta<1$. The range for the parameter $\eta$ was selected using the experimental results 


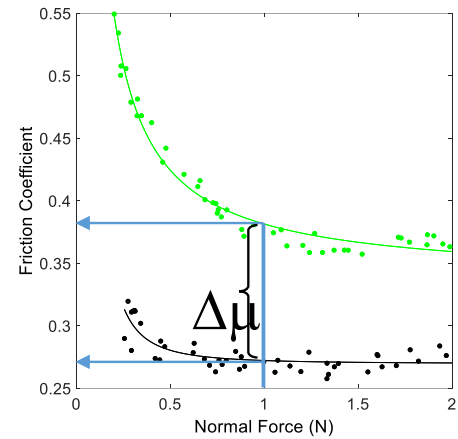

a)

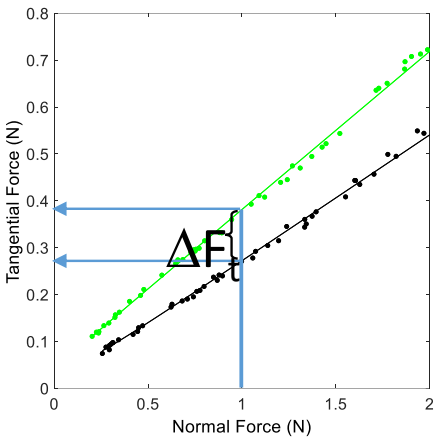

b)

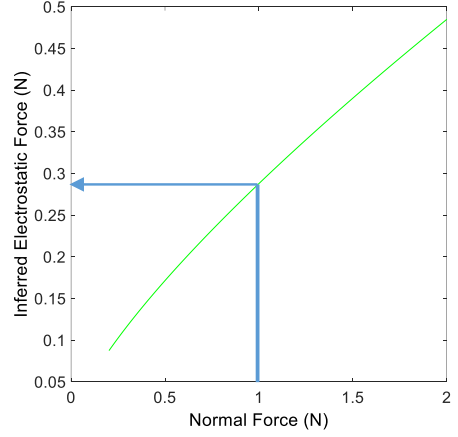

c)

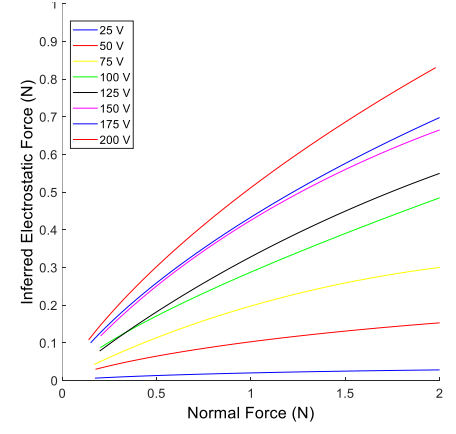

d)

Fig. 5. a) Friction coefficient and b) tangential force as a function of normal force for the experimental conditions of EV $=$ OFF and EV $=$ ON (100 Volts). c) Electrostatic force was inferred from the curves given in (a) and (b). d) Inferred electrostatic force as a function of normal force for all the voltage amplitudes tested in this study.

reported in [15].

The optimization routine estimated the effective elastic modulus as $E^{*}=35.00 \mathrm{kPa}$ and interfacial shear strength as $\tau_{0}=10.64 \mathrm{kPa}$. The estimated effective modulus is inline with the experimental values obtained in the earlier studies. Dzidek et al. [12] argued that the rate of increase
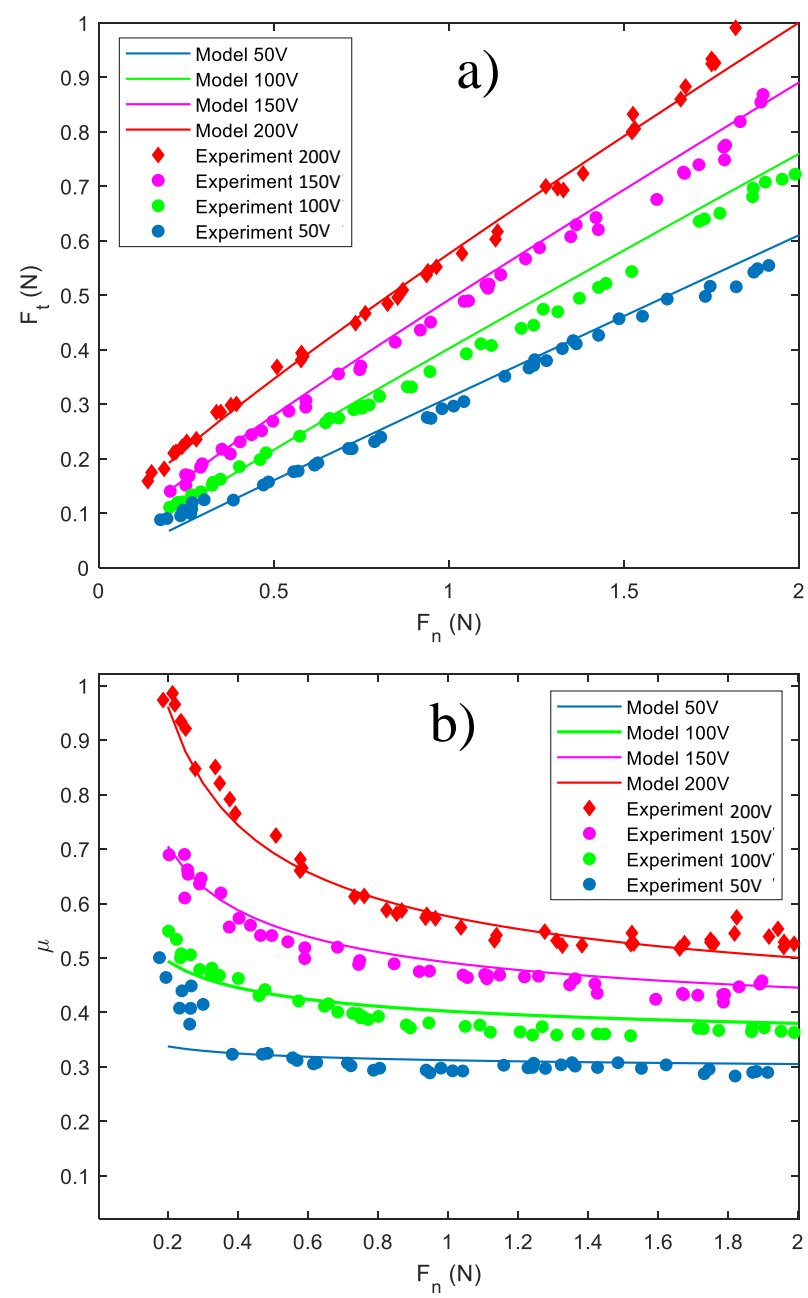

Fig. 6. a) Tangential force versus normal force and b) friction coefficient versus normal force. The solid lines represent the optimized model output for input voltages of 50, 100, 150, 200 Volts. in elastic modulus of fingerpad is small for compression up to $0.9 \mathrm{~mm}$, and estimated the modulus as approximately $E \approx 40 \mathrm{kPa}$. Cornuault et al. [25] estimated the average elastic modulus of fingerpad for a group of male and female subjects as $E=59 \pm 13 k P a$ and $82 \pm 20 k P a$, respectively. Using a robotic platform and imaging system, Delhaye et al. [26] collected data from 4 subjects and estimated the effective elastic modulus as $E^{*} \approx 30,40$, $60 \mathrm{kPa}$ for the normal loads of $0.5,1,2 N$, respectively. Similarly, the value estimated for $\tau_{0}=10.64 \mathrm{kPa}$ through our optimization process is in good agreement with the experimental values reported in the literature for fingerglass interaction. Using the shear stress model of Bowden and Tabor $\left(\tau=\tau_{0}+\alpha p_{e}\right)$, Adams et al. [7] estimated the shear strength as $\tau_{0}=4.6 \mathrm{kPa} \mathrm{kPa}$ for wet skin sliding against a glass probe for contact pressures varying between 1 and $17 k P a$. Hendricks and Franklin [27] followed the same model and reported that $\tau_{0}$ varies between 0.21-2.1 $k P a$ for the dry skin on the forearm. Again, using the same model, Derler et al. [28] fit a limiting curve to experimental data of friction coefficient versus apparent contact pressure (such that all the experimental data points lie below this curve) using the value of $\tau_{0}=13.3 \mathrm{kPa}$. In their study, the intrinsic shear strength $\tau_{0}$ was assumed to be equal to the shear modulus $G$, which was estimated from $E=3 G$ by assuming bulk elastic modulus of finger skin as $E=40 \mathrm{kPa}$. Our model also estimated the ratio of real contact area to the apparent one, $\beta$. When there is no electroadhesion, this ratio is equal to $\beta=\beta_{0}$, which was estimated by optimization as 0.005 (i.e. only $0.5 \%$ of the apparent area). However, it was found to increase nonlinearly from $\beta=$ 0.02 to $0.16(2-16 \%$ of the apparent area) as the input voltage amplitude is increased from 50 to 200 Volts with increments of 50 Volts (Fig. 7c). These values are also in good agreement with the results of the recent experimental studies utilizing more sophisticated contact area measurements. In the earlier studies, the real area of contact has been taken as the contact area of finger ridges $\left(A_{\text {ridge }}\right)$, which is obviously smaller than the apparent contact area $\left(A_{\text {app }}\right)$ estimated by the fingerprint images using the boundaries of finger pad in contact with a counter surface. Warman and Enno [29] investigated the relations between $A_{\text {ridge }}$, $A_{a p p}$, and normal force for fingerpad using ink print and 
a)

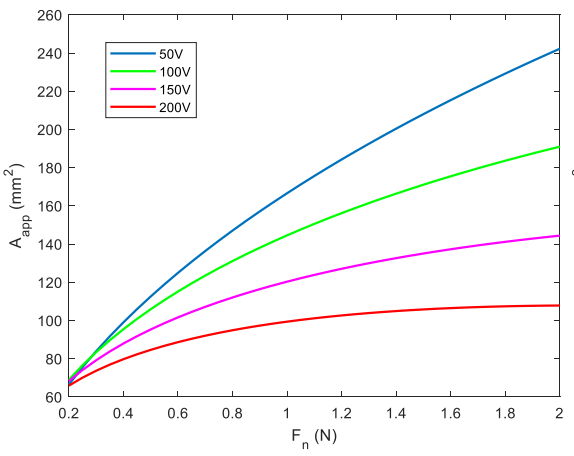

b)

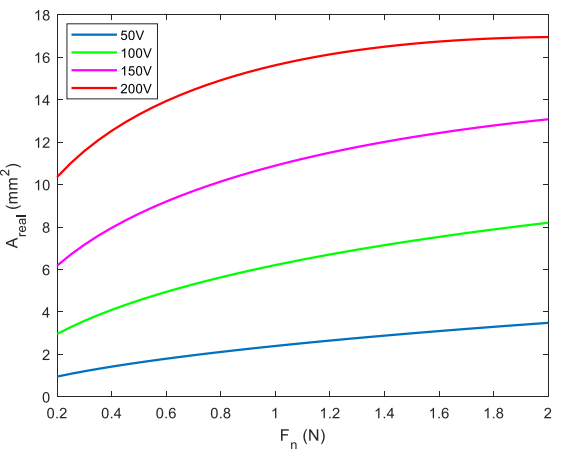

c)

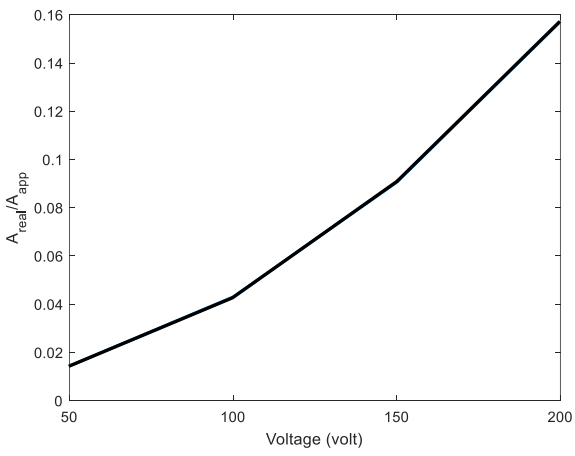

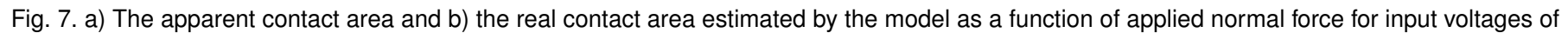
$50,100,150,200$ Volts. c) The ratio of real contact area to apparent contact area as a function of input voltages.

reported that $A_{\text {ridge }}$ is proportional to the power of normal force and the power term varies between 0.68 and 0.96 . They also found that $A_{a p p}$ is linearly correlated with $A_{\text {ridge }}$ and the ratio of $A_{\text {ridge }} / A_{\text {app }}$ is around 0.7. Tomlinson et al. [30] also reported that the contact area of the finger increased with load following a linear relationship, and the ratio of the ridges area to the total area is between 0.38 and 0.5 . On the other hand, Childs and Henson [31] found that, as the normal force is increased, the increase in $A_{\text {ridge }}$ is higher than that of $A_{a p p}$, and reported the ratio of $A_{\text {ridge }} / A_{a p p}$ as $0.12,0.23$, and 0.34 for the normal forces of $0.41,0.88$, and $1.77 N$. Soneda and Nakano [32] also reported that $A_{\text {ridge }}$ and $A_{a p p}$ are proportional to the power of normal force with the power terms of 0.68 and 0.52 respectively. On the other hand, if the Persson's multi-scale contact theory is considered [5], [6], $A_{\text {real }}$ can be significantly smaller than $A_{\text {ridge }}$ since only the finger asperities at finer resolution make contacts with the surface during sliding. In fact, more recently, Bochereau et al. [33] collected data by using more advanced imaging techniques and reported that the ratio of real area to apparent area varies approximately from 0.05 to 0.3 for the normal forces varying from 0.5 to $2 \mathrm{~N}$ respectively.

In our recent studies [3], [4], the surface roughness of fingerpad was taken into account for the calculation of real contact area using Persson's multi-scale contact theory, which causes finger asperities at different length scales make contacts with the surface due to attractive electrostatic forces, leading to an increase in real contact area. Our model in this study does not take surface roughness and also the variation in air gap thickness into account. We estimated the average thickness of the airgap as $d_{\text {air }}=190 \mathrm{~nm}$. The earlier modeling studies suggested that the air gap should be less than $1 \mu \mathrm{m}$ [3], [34], [35].

We should also point out that our experimental design and measurements do not take into account the variations in mechanical and physicochemical properties of the fingerpad skin, such as morphology, elasticity, hydration, and moisture. Due to the limitations in our set-up (normal force was controlled manually) and the large number of trials, we opted to conduct the experiments with a single and well-trained subject. However, as reviewed by Derler et al. [28], the material properties of human skin show large variations depending on the measurement device, anatomical site, hydration level, age, individual morphology, and finger skin is no exception. As an example, the hydration level in finger skin is known to reduce its elasticity, causing an increase in contact area and thus the tangential frictional force. In our future studies, using the proposed model, we will explore the effects of variations in mechanical and physicochemical properties of fingerpad on the tangential forces acting on it during sliding.

\section{ACKNOWLEDGMENTS}

C.B. acknowledges the financial support provided by TUBITAK under the contract number of 117E954. O.S. acknowledges the doctoral fellowship support provided by TUBITAK under BIDEB-2211 program.

\section{References}

[1] T. Vodlak, Z. Vidrih, E. Vezzoli, B. Lemaire-Semail, and D. Peric, "Multi-physics modelling and experimental validation of electrovibration based haptic devices," Biotribology, vol. 8, pp. 12-25, 2016.

[2] C. D. Shultz, M. A. Peshkin, and J. E. Colgate, "On the electrical characterization of electroadhesive displays and the prominent interfacial gap impedance associated with sliding fingertips," in 2018 IEEE Haptics Symposium. IEEE, 2018, pp. 151-157.

[3] M. Ayyildiz, M. Scaraggi, O. Sirin, C. Basdogan, and B. N. Persson, "Contact mechanics between the human finger and a touchscreen under electroadhesion," Proceedings of the National Academy of Sciences, vol. 115, no. 50, pp. 12668-12 673, 2018.

[4] O. Sirin, M. Ayyildiz, B. Persson, and C. Basdogan, "Electroadhesion with application to touchscreens," Soft Matter, vol. 15, no. 8, pp. 1758-1775, 2019.

[5] B. N. Persson, "Theory of rubber friction and contact mechanics," The Journal of Chemical Physics, vol. 115, no. 8, pp. 3840-3861, 2001.

[6] B. N. Persson, "Contact mechanics for randomly rough surfaces," Surface Science Reports, vol. 61, no. 4, pp. 201-227, 2006.

[7] M. J. Adams, B. J. Briscoe, and S. A. Johnson, "Friction and lubrication of human skin," Tribology Letters, vol. 26, no. 3, pp. 239-253, 2007.

[8] K. L. Johnson, K. Kendall, and A. Roberts, "Surface energy and the contact of elastic solids," Proceedings of the Royal Society of London A: Mathematical and Physical Sciences, vol. 324, no. 1558, pp. 301313, 1971.

[9] T. Yamamoto and Y. Yamamoto, "Dielectric constant and resistivity of epidermal stratum corneum," Medical and Biological Engineering, vol. 14, no. 5, pp. 494-500, 1976.

[10] J. van Kuilenburg, M. A. Masen, and E. van der Heide, "A review of fingerpad contact mechanics and friction and how this affects tactile perception," Proceedings of the Institution of Mechanical Engineers, Part J: Journal of Engineering Tribology, vol. 229, no. 3, pp. 243-258, 2015. 
[11] V. L. Popov and M. Hess, "Voltage induced friction in a contact of a finger and a touchscreen with a thin dielectric coating," arXiv preprint arXiv:1805.08714, 2018.

[12] B. M. Dzidek, M. J. Adams, J. W. Andrews, Z. Zhang, and S. A. Johnson, "Contact mechanics of the human finger pad under compressive loads," Journal of The Royal Society Interface, vol. 14, no. 127, p. 20160935, 2017.

[13] C. Pailler-Mattei, S. Nicoli, F. Pirot, R. Vargiolu, and H. Zahouani, "A new approach to describe the skin surface physical properties in vivo," Colloids and Surfaces B: Biointerfaces, vol. 68, no. 2, pp. 200-206, 2009.

[14] M. Spinner, A. B. Wiechert, and S. N. Gorb, "Sticky fingers: Adhesive properties of human fingertips," Journal of Biomechanics, vol. 49, no. 4, pp. 606-610, 2016.

[15] R. Sahli, G. Pallares, C. Ducottet, I. B. Ali, S. Al Akhrass, M. Guibert, and J. Scheibert, "Evolution of real contact area under shear and the value of static friction of soft materials," Proceedings of the National Academy of Sciences, vol. 115, no. 3, pp. 471-476, 2018.

[16] O. Sirin, A. Barrea, P. Lefèvre, J.-L. Thonnard, and C. Basdogan, "Fingerpad contact evolution under electrovibration," Journal of the Royal Society Interface, vol. 16, no. 156, p. 20190166, 2019.

[17] V. L. Popov and A. V. Dimaki, "Friction in an adhesive tangential contact in the coulomb-dugdale approximation," The Journal of Adhesion, vol. 93, no. 14, pp. 1131-1145, 2017.

[18] A. Savkoor and G. Briggs, "The effect of tangential force on the contact of elastic solids in adhesion," Proceedings of the Royal Society of London A: Mathematical and Physical Sciences, vol. 356, no. 1684, pp. 103-114, 1977.

[19] J. F. Waters and P. R. Guduru, "Mode-mixity-dependent adhesive contact of a sphere on a plane surface," Proceedings of the Royal Society A: Mathematical, Physical and Engineering Sciences, vol. 466, no. 2117, pp. 1303-1325, 2009.

[20] F. Bowden and D. Tabor, "The friction and lubrication of solids," Oxford University Press, 1954.

[21] M. J. Adams, S. A. Johnson, P. Lefèvre, V. Lévesque, V. Hayward, T. André, and J.-L. Thonnard, "Finger pad friction and its role in grip and touch," Journal of The Royal Society Interface, vol. 10, no. 80, p. 20120467, 2013.

[22] M. Ruths and J. Israelachvili, "Surface forces and nanorheology of molecularly thin films," Springer Handbook of Nanotechnology, pp. 859-924, 2007.

[23] D. J. Meyer, M. A. Peshkin, and J. E. Colgate, "Fingertip friction modulation due to electrostatic attraction," in 2013 IEEE World Haptics Conference. IEEE, 2013, pp. 43-48.

[24] S. Derler, L.-C. Gerhardt, A. Lenz, E. Bertaux, and M. Hadad, "Friction of human skin against smooth and rough glass as a function of the contact pressure," Tribology International, vol. 42, no. 11-12, pp. 1565-1574, 2009.

[25] P.-H. Cornuault, L. Carpentier, M.-A. Bueno, J.-M. Cote, and G. Monteil, "Influence of physico-chemical, mechanical and morphological fingerpad properties on the frictional distinction of sticky/slippery surfaces," Journal of The Royal Society Interface, vol. 12, no. 110, p. 20150495, 2015.

[26] B. Delhaye, P. Lefevre, and J.-L. Thonnard, "Dynamics of fingertip contact during the onset of tangential slip," Journal of The Royal Society Interface, vol. 11, no. 100, p. 20140698, 2014.

[27] C. Hendriks and S. Franklin, "Influence of surface roughness, material and climate conditions on the friction of human skin," Tribology Letters, vol. 37, no. 2, pp. 361-373, 2010.

[28] S. Derler and L.-C. Gerhardt, "Tribology of skin: review and analysis of experimental results for the friction coefficient of human skin," Tribology Letters, vol. 45, no. 1, pp. 1-27, 2012.

[29] P. H. Warman and A. R. Ennos, "Fingerprints are unlikely to increase the friction of primate fingerpads," Journal of Experimental Biology, vol. 212, no. 13, pp. 2016-2022, 2009.

[30] S. Tomlinson, R. Lewis, X. Liu, C. Texier, and M. Carré, “Understanding the friction mechanisms between the human finger and flat contacting surfaces in moist conditions," Tribology Letters, vol. 41, no. 1, pp. 283-294, 2011.

[31] T. Childs and B. Henson, "Human tactile perception of screenprinted surfaces: self-report and contact mechanics experiments," Proceedings of the Institution of Mechanical Engineers, Part J: Journal of Engineering Tribology, vol. 221, no. 3, pp. 427-441, 2007.

[32] T. Soneda and K. Nakano, "Investigation of vibrotactile sensation of human fingerpads by observation of contact zones," Tribology International, vol. 43, no. 1-2, pp. 210-217, 2010.
[33] S. Bochereau, B. Dzidek, M. Adams, and V. Hayward, "Characterizing and imaging gross and real finger contacts under dynamic loading," IEEE Transactions on Haptics, vol. 10, no. 4, pp. 456-465, 2017.

[34] B. Persson, "The dependency of adhesion and friction on electrostatic attraction," The Journal of Chemical Physics, vol. 148, no. 14, p. 144701, 2018.

[35] A. Almqvist, C. Campana, N. Prodanov, and B. Persson, "Interfacial separation between elastic solids with randomly rough surfaces: comparison between theory and numerical techniques," Journal of the Mechanics and Physics of Solids, vol. 59, no. 11, pp. 2355-2369, 2011.

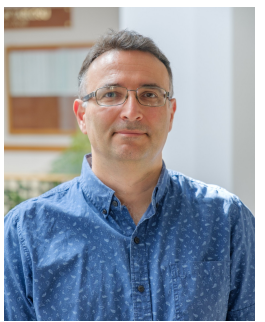

Cagatay Basdogan received the Ph.D. degree in mechanical engineering from Southern Methodist University, in 1994. He is a faculty member both in the mechanical engineering and the computational sciences and engineering programs at Koc University, Istanbul, Turkey. $\mathrm{He}$ is also the director of the Robotics and Mechatronics Laboratory, Koc University. Before joining Koc University, he worked at NASAJPL/Caltech, MIT, and Northwestern University Research Park. His research interests include haptic interfaces, robotics, mechatronics, biomechanics, medical simulation, computer graphics, and multi-modal virtual environments. $\mathrm{He}$ is currently the associate editor in chief of the IEEE Transactions on Haptics and serves on the editorial boards of the IEEE Transactions on Mechatronics, Presence: Teleoperators and Virtual Environments, and Computer Animation and Virtual Worlds journals. In addition to serving in programme and organizational committees of several haptics conferences, he chaired the IEEE World Haptics Conference in 2011.

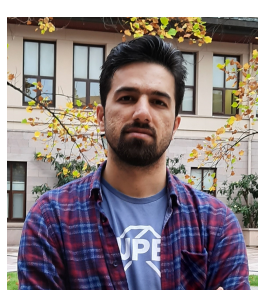

M.Reza Alipour Sormoli is a $\mathrm{PhD}$ student in Mechanical Engineering Department of Koc University in Istanbul, Turkey. He received his BSc degree from University of Tabriz in 2014 and MSc degree from Amirkabir University of Technology (Tehran Polytechnic) in 2017, all in mechanical engineering. His research interests are haptics, mechatronics, control and dynamics, and contact mechanics.

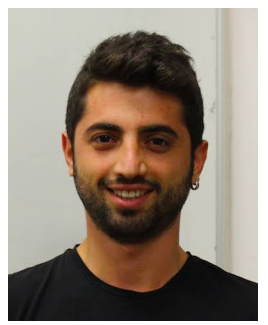

Omer Sirin received his Ph.D. degree from Mechanical Engineering Department of Koc University in Istanbul, Turkey in 2019. He was awarded the prestigious TUBITAK BIDEB fellowship for his doctoral studies. He received his BSc degree in Mechatronics Engineering from Bahcesehir University in 2012 and his MSc degree in Biomedical Engineering from Cleveland State University in 2013. His research interests are haptics, mechatronics, and contact mechanics. 